\title{
Planejamento para a Sustentabilidade: lições aprendidas a partir do projeto BEAUTY and HEALTH, Carolina do Norte, EUA
}

\author{
Planning for the Sustainability: lessons learned from project BEAUTY and \\ HEALTH, North Caroline, USA
}

\author{
Planificación para la Sustentabilidad: lecciones aprendidas a partir del \\ Proyecto BEAUTY and HEALTH, Carolina del Norte, EUA
}

\author{
Recebido: 11/05/2013
}

Aprovado: 23/02/2014

\author{
Laura Ann Linnan ${ }^{1}$
}

Este artigo tem como objetivo relatar a experiência de formação de esteticistas licenciados para promover saúde no salão de beleza e descreve os recursos necessários dentro da comunidade em geral, os fatores dentro das organizações, o design de programas e os fatores de implementação, bem como outros componentes essenciais do modelo RE-AIM, que foram considerados no "planejamento" para a sustentabilidade de um componente de intervenção eficaz do projeto BEAUTY and Health (Beleza e Saúde) no Estado da Carolina do Norte, EUA. A sustentabilidade de programas de promoção da saúde eficazes merece consideração especial dada à escassez de literatura sobre o tema, bem como para a redução de custos de manutenção de importantes benefícios críticos para a saúde e os esforços de capacitação.

Descritores: Promoção da saúde; Desenvolvimento de programas; Comportamentos saudáveis.

The objective of this article is to describe the training of licensed cosmetologists to promote health in beauty shops and the key factors that were considered for "planning for sustainability" of the intervention. Furthermore, describes features within the broader community, factors within organizations, and program design and implementation factors, as well as key components of the RE-AIM framework, that were considered when "planning" for sustainability of an effective intervention component of the North Carolina BEAUTY and Health Project. The sustainability of effective health promotion programs warrants special consideration given the paucity of literature on this topic, as well as the important cost savings of maintaining critical health benefits and capacity-building efforts.

Descriptors: Health promotion; Program development; Health behavior.

Este artículo tiene como objetivo relatar la experiencia de formación de esteticistas licenciados para promover salud en el salón de belleza y describe los recursos necesarios dentro de la comunidad en general, los factores dentro de las organizaciones, el diseño de programas y los factores de implementación, así como otros componentes esenciales del modelo RE-AIM, que fueron considerados en la "planificación" para la sustentabilidad de un componente de intervención eficaz del proyecto BEAUTY and Health (Belleza y Salud) en el Estado de Carolina del Norte, EUA. La sustentabilidad de programas de promoción de la salud eficaces merece consideración especial dada la escasez de literatura sobre el tema, así como para la reducción de costos de mantenimiento de importantes beneficios críticos para la salud y los esfuerzos de capacitación.

Descriptores: Comportamientos saludables; Desarrollo de programas; Promoción de la salud.

\footnotetext{
${ }^{1}$ Educadora em Saúde. Especialista em Educação em Saúde. Mestre em Educação em Saúde Pública. Doutora em Saúde e Comportamento Social. Professora do Mestrado em Saúde Pública e do Doutorado em Saúde Comportamental e Educação em Saúde do Departamento de Comportamento em Saúde e Educação em Saúde da Universidade da Carolina do Norte de Chapel Hill, EUA. linnan@email.unc.edu
} 


\section{INTRODUÇÃO}

A sustentabilidade de programas eficazes de promoção da saúde merece consideração crítica. Primeiro, o custo de criação e desenvolvimento de programas são altos, de modo que se um programa tem resultados positivos para a saúde, manter este programa (ao invés de custear outra produção) faz todo sentido.

Em segundo lugar, a sustentabilidade ajuda programas eficazes a perdurar além dos ciclos de financiamento iniciais. Se um programa é eficaz, (por exemplo, se os benefícios destinados são realizados), então a perpetuação desses benefícios é um resultado altamente desejável.

Terceiro, programas eficazes muitas vezes produzem inesperados impactos benéficos tais como empregabilidade, capacitação e confiança. Por último, há escassez de literatura sobre a sustentabilidade dos programas de saúde pública, com algumas exceções ${ }^{1-5}$. O estudo da sustentabilidade está em sua infância, momento no qual a necessidade de identificar e manter programas efetivos é imensa.

Este artigo oferece uma definição funcional de sustentabilidade e aplicação dos principais aspectos do modelo de ShediacRizkallah \& Bone ${ }^{1}$ relacionados a uma oficina de capacitação eficaz, que emergiu como digna de se "sustentar" por meio de programas de Educação Continuada (EC). Esta oficina de formação foi desenvolvida como parte do "Projeto BEAUTY and Health da Carolina do Norte (NC)" (conhecido como "BEAUTY Project").

Sustentabilidade é descrita como "o grau em que uma intervenção baseada em evidências pode produzir efeitos pretendidos durante um extenso período de tempo depois que suporte externo de agências de financiamento é encerrado"1. Vários aspectos desta definição são notáveis. Primeiro, concentra-se em intervenções "baseadas em evidências", presumindo que algumas evidências de efetividade estão disponíveis através da revisão de literatura, em compêndios, tais como o Guia de Serviços Preventivos do Centers for Disease Control and Prevention (CDC) dos Estados Unidos, as Revisões Colaborativas do Cochrane, e outras.

Segundo, esta definição descreve que as intervenções eficazes devem oferecer benefícios "durante um longo período de tempo após o financiamento externo... ser encerrado". Esta definição não impõe um prazo específico e definido para a produção de efeitos reconhecendo que as intervenções frequentemente estão em diferentes fases de execução quando o financiamento externo é concluído. E que, alguns efeitos acontecem rapidamente, enquanto outros podem não vir à tona até muito mais tarde. Além disso, se os programas eficazes são encerrados prematuramente, podem trazer complicações tais como a quebra de relacionamentos e desconfiança ou apatia dos envolvidos com os programas, de modo que os esforços futuros dentro de um determinado grupo ou comunidade são menos propensos a ser bem sucedidos.

Esta definição também aponta que a sustentabilidade não é uma proposta de "tudo ou nada", mas sim um processo. Os efeitos de sustentabilidade podem incluir o potencial de se tornarem programas efetivos em rotinas institucionalizadas dentro de um sistema ou organização; o potencial de programas continuarem ou ampliarem seus benefícios para a saúde destinados aos beneficiários; e/ou o potencial de programas ajudarem a construir ou melhorar a capacidade dentro de uma organização ou grupo. Esta tríade feliz de benefícios ocorreu com um programa de atividade física, o Programa de Saúde do Coração Pawtucket, desenvolvido em meados da década de 1980, como parte de um grande estudo de intervenção para doenças cardiovasculares ${ }^{6}$, que foi transferido com sucesso para o Young Men's Christian Association (YMCA) local.

Os membros do YMCA foram capacitados para executar o programa de atividade física acrescentado ao cardápio de 
atividades ofertadas. 0 YMCA recrutou novos membros, recebeu treinamento adicional para o seu pessoal (maior capacidade), acrescentou um programa bem-sucedido ao seu menu de ofertas de atividade física (institucionalização) e estendeu seu alcance para a comunidade (benefícios de saúde estendidos).

Assim, medições de sustentabilidade devem começar no início do processo de planejamento e continuar ao longo do percurso de modo que os programas possam ser mantidos ao longo do tempo. Este artigo tem como objetivo relatar a experiência de formação de esteticistas licenciados para promover saúde no salão de beleza e o processo de "planejamento para a sustentabilidade" da intervenção.

\section{DESCREVENDO A EXPERIÊNCIA Planejamento para a Sustentabilidade: um exemplo}

0 conceito de "planejamento para a sustentabilidade" aqui considerado utiliza a estrutura conceitual proposta por ShediacRizkallah \& Bone (Figura 1$)^{1}$. A Tabela 1 resume as linhas- guias-chaves usadas para descrever fatores do ambiente da comunidade em geral; fatores dentro do ambiente organizacional, concepção do projeto e os fatores de implementação, que se acredita poder influenciar a sustentabilidade do programa.

\section{Contexto - 0 Projeto North Carolina BEAUTY and Health}

Desde o ano 2000, uma equipe interdisciplinar de pesquisadores da Universidade da Carolina do Norte Chapel Hill tem trabalhado em parceria com os proprietários de salões de beleza e barbearias, esteticistas/barbeiros e seus clientes para promover saúde dentro desses importantes locais da comunidade ${ }^{7,8}$.

Começando com uma série de pesquisas sobre estudos de formação para salões de beleza, a equipe de pesquisa descobriu que:
1) os salões de beleza estão localizados em todas as comunidades - grande / pequena, urbana / rural;

2) os cabeleireiros muitas vezes têm uma relação única e confiável com seus clientes;

3) os proprietários de salões de beleza e cabeleireiros foram parceiros ávidos na promoção da saúde ${ }^{9,10} \mathrm{e}$;

4) os clientes estavam dispostos a receber informações de saúde de seus cabeleireiros $^{11}$.

Oficinas de formação para cabeleireiros foram um componente-chave do Projeto de intervenção NC BEAUTY and Health (Trazendo educação e compreensão para Você). Resultados iniciais da pesquisa revelaram que os cabeleireiros podem ser treinados para entregar mensagens de saúde fundamentais para seus clientes e que o seu conhecimento e autoeficácia para entregar estas mensagens foi melhorada com a participação na oficina; além disso, os clientes ficaram satisfeitos em receber informações de saúde de seus cabeleireiros $^{12}$.

Seis oficinas diferentes de formação para cabeleireiros sobre temas relacionados com a atividade física, alimentação saudável, peso e rastreamento de câncer foram implementadas com sucesso durante o Projeto BEAUTY12. Além disso, tranformouse uma das oficinas originais de formação do Projeto BEAUTY em uma nova oficina de formação de educação continuada (EC) para novos cabeleireiros licenciados em NC que seriam sustentadas além do período de financiamento da pesquisa usando o modelo "planejamento para a sustentabilidade".

\section{Fatores Comunitários}

0 Conselho Estadual de Arte Cosmética da Carolina do Norte aprovou regulamentos que exigem que todos os cabeleireiros e esteticistas devem completar 24 créditos de EC a cada 3 anos a fim de manter a sua licença em cosmetologia. Esta exigência foi instituída quando subsídio que financiava o estudo em questão estava terminando. 
Tabela 1: Considerações para Sustentabilidade do Projeto BEAUTY \& Health, Carolina do Norte, EUA.

\begin{tabular}{|c|c|}
\hline & $\begin{array}{l}\text { Projeto NC BEAUTY and Health -Oficinas de Educação Continuada para } \\
\text { Cosmetologistas Licenciados }\end{array}$ \\
\hline $\begin{array}{l}\text { Fatores Gerais do } \\
\text { Ambiente Social, } \\
\text { Político, Regulatório e } \\
\text { Econômico }\end{array}$ & $\begin{array}{l}\text { O Conselho Estadual de Arte Cosmética da Carolina do Norte exige que } \\
\text { cosmetologistas/cabeleireiros e esteticistas obtenham } 24 \text { horas de crédito de } \\
\text { educação continuada a cada } 3 \text { anos, a fim de manter o licenciamento (política). Os } \\
\text { membros do Conselho Consultivo do BEAUTY e cabeleireiros participantes } \\
\text { recomendaram que as oficinas de capacitação para os cabeleireiros do estudo } \\
\text { BEAUTY fossem adaptadas e depois submetidas à aprovação de crédito de EC } \\
\text { (participação da comunidade). Alguns cursos estavam disponíveis quando esta } \\
\text { nova resolução entrou em vigor e os custos do curso de EC eram muitas vezes } \\
\text { impeditivos (econômica). }\end{array}$ \\
\hline $\begin{array}{l}\text { - Processo de } \\
\text { negociação do projeto } \\
\text { - Efetividade do projeto } \\
\text { - Duração do projeto } \\
\text { - Financiamento do } \\
\text { projeto } \\
\text { - Capacitação }\end{array}$ & $\begin{array}{l}\text { Seis oficinas de formação de quatro horas para cabeleireiros foram criadas como } \\
\text { parte do Projeto BEAUTY. Cabeleireiros que participaram da pesquisa pediram aos } \\
\text { membros da equipe de pesquisa para transformar essas oficinas em oportunidades } \\
\text { de EC (negociação). Evidências sugerem que os cabeleireiros que participaram das } \\
\text { oficinas melhoraram sua auto-eficácia para promover mensagens essenciais de } \\
\text { saúde e estavam dispostos a participar de treinamentos futuros (12) (efetividade) } \\
\text { e, observou-se que os clientes que relataram falarem regularmente com seus } \\
\text { cabeleireiros relataram maior mudança de comportamentos saudáveis (12) } \\
\text { (efetividade). As oficinas de formação originais eram de } 4 \text { horas, e seis oficinas } \\
\text { diferentes foram desenvolvidas. Considerando que cursos de EC para cabeleireiros } \\
\text { licenciados são normalmente oferecidos em meio ou um dia inteiro de oficina, } \\
\text { decidiu-se oferecer um curso EC de oito horas inicialmente (duração). Após } \\
\text { pesquisa de mercado com os cabeleireiros licenciados, ofertamos as oficinas de } \\
\text { formação a um baixo custo (US } \$ 40 \text { para oito créditos EC) em relação a outros } \\
\text { cursos de formação de EC este valor conseguia cobrir as despesas para oferecer a } \\
\text { oficina de formação, porque apenas um dos três instrutores recebeu pagamento } \\
\text { por suas contribuições, enquanto os outros dois tiveram serviços "em espécie" em } \\
\text { retorno (financiamento). Vários indivíduos foram envolvidos nas seis oficinas de } \\
\text { capacitação oferecidos como parte do projeto BEAUTY. Nas oficinas de formação de } \\
\text { EC, dois membros da equipe de pesquisa e um membro do Conselho Estadual que } \\
\text { também trabalhava como diretor de uma escola de cosmetologia e serviram como } \\
\text { instrutores. Este acordo reflete a parceria entre pesquisadores da UNC e da } \\
\text { indústria de salão de beleza, assegurando conhecimentos necessários à tarefa. } \\
\text { Apesar de não ser complicado, requisitos da EC obrigavam que } 50,0 \% \text { do programa } \\
\text { abrangessem tópicos relacionados à cosmetologia. A oficina de EC foi originalmente } \\
\text { concebida para uma sessão de oito horas, porque os cabeleireiros licenciados } \\
\text { solicitaram esta opção; no entanto, é possível oferecer uma versão quatro horas / } \\
\text { quatro créditos dessa oficina também. }\end{array}$ \\
\hline $\begin{array}{lr}\text { Fatores do âmbito } \\
\text { Organizacional } \\
\text { Comunitário- } \\
\text { Defensores chaves / } \\
\text { liderança do programa- } \\
\text { Integração r rom } \\
\text { programas / } \\
\text { existentes- } \\
\text { Institucional }\end{array}$ & $\begin{array}{l}\text { Defensores chaves do programa incluem membros da equipe de pesquisa, do } \\
\text { Conselho Consultivo e do Conselho Estadual de Arte Cosmética que aprovaram este } \\
\text { curso para receber créditos de EC. Além disso, os participantes da pesquisa original } \\
\text { do estudo (mais de } 40 \text { proprietários de salão de beleza e mais de } 70 \text { estilistas } \\
\text { licenciados) também serviram como apoiadores do programa ajudando a promover } \\
\text { esta oficina. Esta oficina de EC é disponibilizada como parte do menu do Conselho } \\
\text { Estadual de Arte Cosmética de NC e tornou-se uma operação verticalmente } \\
\text { integrada. Para atender aos requisitos estaduais de EC, os objetivos da oficina de } \\
\text { capacitação da EC são especificados e consistentes com a missão da EC. Ambos - } \\
\text { UNC e Conselho Estadual de Arte Cosmética - tem força institucional e podem dar } \\
\text { suporte a novos programas na medida em que o menu de ofertas continua a } \\
\text { crescer. }\end{array}$ \\
\hline
\end{tabular}


Figura 1: Adaptação*do Modelo Shediac-Rizkallah \& Bone ${ }^{1}$ para Sustentabilidade de Programa.

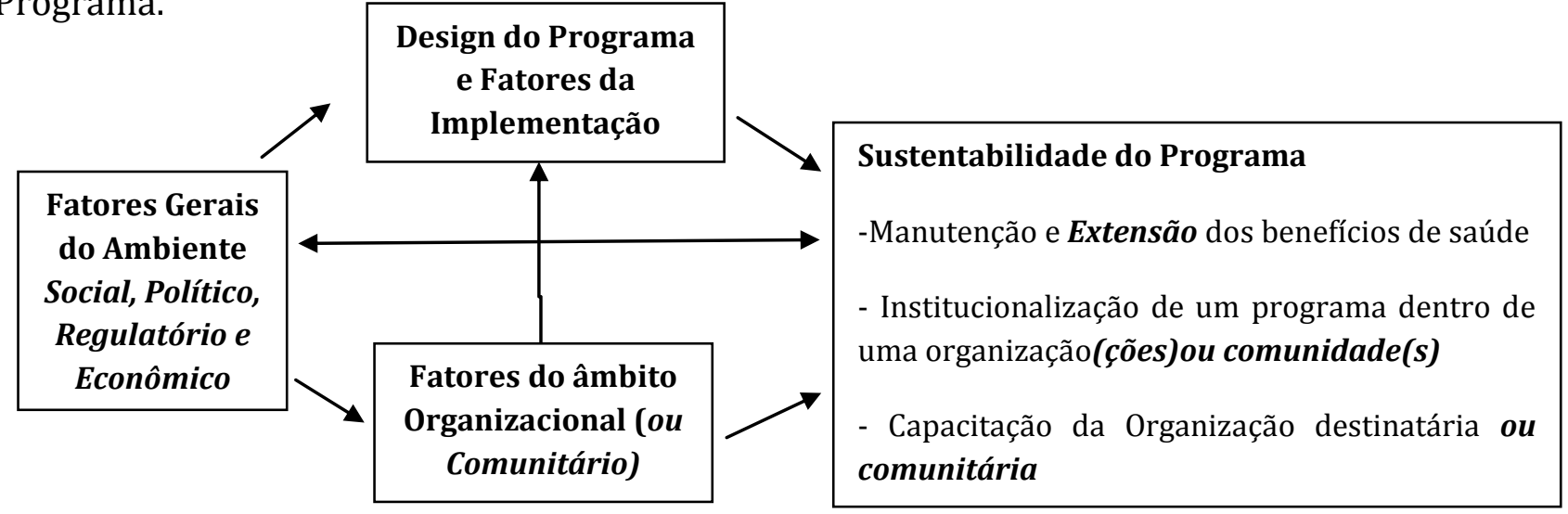

*Os itens em negrito e itálico foram adaptações do modelo original1.

Recebeu-se então a aprovação pelo Conselho Estadual de oferecer uma oficina de oito créditos de EC para estilistas licenciados com base nas oficinas de formação bem sucedidas que já se tinha oferecido como parte do financiamento da pesquisa. Assim, as condições políticas, regulamentares e econômicas foram favoráveis para o lançamento de um curso de EC para cabelereiros licenciados com base em pesquisas sobre oficinas de treinamento.

Realizou-se então uma parceria com cabeleireiros locais e donos de salões para ajudar a promover esta oficina e os participantes da pesquisa anterior foram convidados para atender as novas oficinas. Outros membros altamente respeitados do Conselho foram convidados para servir como instrutores no curso aprovado para ajudar a atrair grande variedade de cabeleireiros licenciados.

\section{Desenho do Programa e Fatores Implementação}

Para os fatores relacionados ao desenho do programa, se fez ampla pesquisa de mercado para determinar o tempo, custo e conteúdo das oficinas de EC. 0 projeto BEAUTY inscreveu mais de 70 estilistas e 40 proprietários de salões de beleza para o estudo. Todos foram entrevistados para determinar as suas opiniões e interesse em oficinas de EC. Também foram convidados para fornecerem feedback sobre qualquer aspecto das oficinas de capacitação oferecidas como parte de um estudo maior.

Também se avaliou a efetividade do projeto como por exemplo, efeitos reais incluindo percepções, visibilidade e os efeitos secundários. As oficinas de capacitação piloto revelaram que ambos os cabeleireiros e os clientes ficaram satisfeitos com os treinamentos. Posteriormente, mudou-se o foco da EC para a saúde pessoal dos cabeleireiros.

A duração do programa de EC foi estendida de quatro para oito horas, porque os cabeleireiros relataram que preferiam completar a maioria de seus créditos de EC "de uma vez e em um só lugar". As quatro horas das oficinas do projeto BEAUTY foram facilmente estendidas para oito horas, adicionando $50,0 \%$ do conteúdo que obrigatoriamente deveria ser "relacionado a cosmetologia" para atender aos requisitos da EC. Foram avaliados os custos de desenvolvimento do programa, os custos recorrentes e ainda se planejou uma versão online do curso de formação que foi aprovado para a implementação.

Quanto aos fatores de execução do programa, considerou-se a formação de instrutores (como tipo de pessoal e conhecimentos necessários), maximizando a flexibilidade e minimizando a complexidade. 
Dois membros da equipe de pesquisa e um membro do Conselho Estadual foram os instrutores-chave para esse curso de EC.

Cada um tinha participado no desenvolvimento das oficinas de capacitação do projeto BEAUTY e o membro do Conselho também era o diretor da Escola de Cosmetologia da Faculdade Comunitária do Condado de Guilford. A complexidade de implementar o curso foi minimizada, incluindo partes que foram aplicadas por vídeo. Manuais de treinamento foram desenvolvidos para que novos instrutores, pudessem facilmente implementar seções específicas da oficina.

Fatores do Ambiente Organizacional e/ou Comunitários

As oficinas de capacitação do projeto BEAUTY foram mantidas dentro do menu de cursos de EC oferecidos pelo Conselho Estadual de Arte Cosmética da Carolina do Norte. Um denfensor-chave para o programa ajudou a estabelecer o programa de EC para cabeleireiros licenciados.

Por recomendação dos cabeleireiros e profissionais das escolas de beleza entrevistados, decidiu-se não integrar a formação de cabeleireiros nos requisitos para a formação do aluno / licenciamento de cabeleireiros; mas em vez disso, integrá-lo nos (novos) requisitos de EC. A missão do programa de EC foi inteiramente compatível com as oficinas de treinamento originais do projeto BEAUTY. A força das instituições envolvidas - Universidade da Carolina do Norte e do Conselho Estadual de Arte Cosmética - foi um bom presságio para sustentabilidade em longo prazo ${ }^{13}$.

\section{DISCUSSÃO}

Intervenções baseadas na comunidade que são planejadas para a sustentabilidade tem a melhor chance de manter ou estender benefícios de saúde desejados, capacitar as organizações e comunidades parceiras, e/ou institucionalizar o programa dentro de uma determinada organização ou comunidade.

Em outras palavras, o planejamento ajuda a garantir que os programas eficazes "dêem certo", construam relacionamentos e confiança mútua que podem apoiar a continuidade programática de um curso. Glasgow e colaboradores desenvolveram o modelo original do RE-AIM ${ }^{14}$ para ajudar a planejar e avaliar diferentes intervenções com base em alcance, efetividade, adoção, implementação e manutenção. Hoje, o REAIM tem sido aplicado a uma ampla gama de problemas de saúde e mais de 100 artigos foram publicados sobre o seu uso no planejamento, avaliação e translação de pesquisas para a prática (ver http://www.re-aim.hnfe.vt.edu/about reaim/what is re-aim/index.html) ${ }^{15}$.

A oficina aprovada para a EC de cabeleireiros licenciados foram avaliados ao longo dessas mesmas construções. Especificamente, o alcance ("Reach") mediu o número absoluto, proporção e/ou representatividade dos indivíduos que se inscrevem no programa de EC.

A "Eficácia" foi medida pelo impacto do seminário de formação sobre os resultados principais (como mudança no conhecimento do cabeleireiro, na autoeficácia ou no comportamento) e inclui potenciais efeitos negativos, qualidade de vida e resultados econômicos.

A"Adoção" é o número absoluto, proporção e representatividade das localidades e/ou agentes da intervenção (pessoas que executaram o programa) que estão dispostos a ingressar no programa. No caso das oficinas de EC, a adoção seria o número de grupos (por exemplo, franquias de salões ou escolas de beleza) que estão dispostas a patrocinar e/ou promover as oficinas de EC para os cabeleireiros licenciados.

A" Implementação " refere-se à fidelidade que cada treinador tem ao protocolo original da oficina de capacitação, incluindo a consistência do tempo de execução e o custo da intervenção. No nível individual, a implementação refere-se ao uso dos clientes de cada componente das estratégias de intervenção. 
Por sua vez, a "Manutenção" refere-se à medida que a oficina de EC se torna institucionalizada como parte do menu de ofertas de cursos de EC aceitas pelo Conselho Estadual de Arte Cosmética de NC para obtenção de créditos de EC por cabeleireiros licenciados. Além disso, a manutenção no nível individual refere-se aos efeitos em longo prazo da oficina de EC sobre os resultados em cabeleireiros por seis ou mais meses pós-treinamento. Assim, os fundamentos do RE-AIM são melhor utilizados como parte do processo de planejamento.

Cada um dos fundamentos do RE-AIM deve ser cuidadosamente operacionalizado, monitorado e utilizado como um meio de intensificar os esforços para planejar a sustentabilidade. Especificamente, se a oficina de EC para os cabeleireiros licenciados tem altos níveis de alcance, eficácia, adoção, implementação e manutenção; e, ainda, considera os fatores gerais do ambiente social, do ambiente organizacional ou comunitário; e aborda a concepção do programa e os fatores-chave de implementação, a probabilidade de sustentar o programa vai ser melhorada.

\section{CONCLUSÃO}

Os planejadores não têm como prática articular os fatores citados (RE-AIM) antes do desenvolvimento e/ou implementação de programas. Assim, é comum que somente ao término do financiamento da intervenção inicial é que se começa a pensar em como manter um programa.

Atentar ao RE-AIM e ao planejamento com abordagens de sustentabilidade são necessárias, mas não totalmente suficientes para sustentar efeitos do programa e a construção de capacidade e/ou institucionalização de oficinas de EC.

Denfensores chaves dos programas e lideranças (ou a falta delas) são criticamente importantes para sustentar os efeitos do programa. A capacidade de manter o conteúdo do programa atualizado em face às alterações das informações ou mudanças nos requisitos de credenciamento podem influenciar a longo prazo a sustentabilidade dessas oficinas.

Planejar antecipadamente para a sustentabilidade esclarece muitos fatores importantes, que influenciam a probabilidade de que os benefícios do programa pretendido sejam realizados ao longo do tempo, e mantidos quando o financiamento inicial não esteja mais disponível.

\section{REFERÊNCIAS}

1. Shediac-Rizkallah MC, Bone LR. Planning for the sustainability of community-based health programs: conceptual frameworks and future directions for research, practice and policy. Health Educ Res. 1998; 13(1):87108.

2. Alexander JA, Weiner BJ, Metzger ME, Shortell SM, Bazzoli GJ, Hasnain-Wynia R, et al. Sustainability of collaborative capacity in community health partnerships. Med Care Res Review. 2003; 60(4 Suppl):130S-59S.

3. Scheirer MA. Is sustainability possible? A review and commentary on empirical studies of program sustainability. Am J Eval. 2005; 26(3):320-47.

4. Pluye P, Potvin L, Denis JL. Making public health programs last: conceptualizing sustainability. Eval Program Plann. 2004; 27(2):121-33.

5. Israel BA, Krieger J, Vlahov D, Ciske S, Foley M, Fortin P, et al. Challenges and facilitating factors in sustaining communitybased participatory research partnerships: Lessons learned from the Detroit, New York City and Seattle Urban Research Centers. J Urban Health. 2006; 83(6):1022-40.

6. Carleton RA, Lasater TM, Assaf AR, Feldman HA, McKinlay $S$, et al. The Pawtucket Heart Health Program: community changes in cardiovascular risk 
factors and projected disease risk. Am J Public Health. 1995; 85(6):777-85.

7. Linnan LA, D'Angelo H, Owens-Ferguson $Y$, Thomas S. Health education and community building in African American barbershops and beauty salons: an innovative approach to addressing health disparities. In: Minkler $\mathrm{M}$, editor. Community organizing and community building for health and welfare. $3^{\text {rd }}$ ed. New York: Rutgers Press; 2012.

8. Linnan LA et al. The North Carolina BEAUTY and Health Project: preventing cancer in African American beauty salons. In: Elk $\mathrm{R}$, Landrine $\mathrm{H}$, organizers. Interventions to reduce cancer disparities: research funded by the American Cancer Society. New York: Springer, Inc; 2011.

9. Solomon F, Linnan LA, Wasilewski Y, Lee AM, Katz ML, Yang J, et al. Observational study in ten beauty salons: using formative research results to inform development of the North Carolina BEAUTY and Health Project. Health Educ Behav. 2004; 31(6):790-805.

10. Linnan LA, Kim AE, Wasilewski $Y$, Lee AM, Yang J, Solomon F, et al. Working with licensed cosmetologists to promote health: results from the North Carolina BEAUTY and health pilot study. Prev Med. 2001; 33(6):606-12.

11. Kim K H, Linnan LA, Kulik N, Carlisle V, Enga Z, Bentley $M$, et al. Linking beauty and health among African American women: using focus group results to build culturally and contextually appropriate interventions. J Soc Behav Health Sci. 2007; 1(1):41-59.

12. Linnan LA, Ferguson YO. Beauty salons: a promising health promotion setting for reaching and promoting health among African American women. Health Educ Behav. 2007; 6(37):517-30.

13. Linnan LA, Rose, JM, Carlisle VA, Evenson KR, Mangum A, Hooten E, et al. The North Carolina BEAUTY and Health Project: overview and baseline results. Community Psychol. 2007; 40(2):61-6.

14. Glasgow RE, Vogt TM, Boles SM. Evaluating the public health impact of health promotion interventions: the RE-AIM framework. Am J Public Health. 1999; 89(9):1322-7.

15. Implementation Science: Integrating Science, Practice, Policy. http://www.reaim.hnfe.vt.edu/about_re-aim/what_is_reaim/index.html (citado em 17/02/2014) (atualizado em 01/01/2014).

\section{CONTRIBUIÇÕES}

Laura Ann Linnan foi responsável pela condução do estudo apresentado, bem como pela descrição da experiência de pesquisa e redação do artigo.

\section{AGRADECIMENTOS}

Agradecimentos a American Cancer Society (TURSG-02-190-01-PBP) pelo apoio à esse estudo. 